Buist, K. L., Metindogan, A., Coban, S., Watve, S., Paranjpe, A., Koot, H. M., van Lier, P., Branje, S. J. T., \& Meeus, W. H. J. (2017). Cross-cultural differences in sibling power balance and its concomitants across three age periods. In N. Campione-Barr (Ed.), Power, control, and influence in sibling relationships across development. New Directions for Child and Adolescent Development. 156, 87-104.

\title{
Cross-Cultural Differences in Sibling Power Balance and Its Concomitants Across Three Age Periods
}

\author{
Kirsten L. Buist, Aysegül Metindogan, Selma Coban, Sujala Watve, \\ Analpa Paranjpe, Hans M. Koot, Pol van Lier, Susan J. T. Branje, \\ Wim H. J. Meeus
}

\begin{abstract}
We examined cross-cultural differences in (1) sibling power balance and (2) the associations between sibling power balance and internalizing and externalizing problems in three separate cross-cultural studies (early childhood, late childhood, and adolescence). The early childhood samples consisted of 123 Turkish and 128 Dutch mothers (mean age for children was 4.9 years). In the late childhood samples, self-report data were collected from 124 Indian and 129 Dutch children (mean age 10.9 years). In the adolescent samples self-report data were collected from 165 ethnic Moroccan and 165 ethnic Dutch adolescents (mean age 15.2 years). In all studies, questionnaire data on sibling power imbalance and internalizing and externalizing problems were collected. Results showed only one significant cross-cultural difference in sibling power imbalance: The Indian sample reported more sibling power imbalance than the Dutch. Links between sibling power imbalance and problem behavior were highly similar between the different cultural samples. The only significant difference was a stronger impact of sibling power imbalance on externalizing problems for the Dutch compared to the Turkish sample. Concluding, few cross-cultural differences were found in sibling power imbalance. Across cultures and age groups, more sibling power imbalance was linked to more internalizing and externalizing problems. (C) 2017 Wiley Periodicals, Inc.
\end{abstract}


S ibling relationships have been linked with many positive and negative outcomes (Buist, Deković, \& Prinzie, 2013; Dirks, Persram, Recchia, \& Howe, 2015). One of the key characteristic of the sibling relationship is sibling power balance (Buhrmester, 1992). Sibling power balance reflects the degree to which there is symmetry of power distribution within the sibling dyad (Furman \& Buhrmester, 1985). However, sibling power balance is an understudied subject (Caspi, 2011; Tucker, Updegraff, $\&$ Baril, 2010). This is troubling because the few studies that have looked at sibling power balance have suggested a link with problem behavior (East $\&$ Khoo, 2005). Moreover, much of the knowledge about sibling power balance and its link to psychosocial functioning stems from research conducted in Western countries, often with ethnic majority samples. Because the nature and function of sibling relationships may differ as a function of ethnicity and cultural background (Cicirelli, 1994), it seems important to examine whether sibling power balance and its concomitants show crosscultural differences.

\section{Sibling Power Balance and Psychosocial Functioning}

There are several reasons for expecting a link between sibling power balance and psychosocial functioning. Abuhatoum and Howe (2013) examined the use of sibling power in conflict situations in a White Canadian sample and found that power imbalance between siblings was associated with aggressive and coercive behavior between siblings. Negative sibling interactions have consistently been linked to internalizing and externalizing problems (Buist et al., 2013). Children and adolescents may become anxious and depressed from being dominated by their sibling or from being exposed to negative sibling interactions. Additionally, they may generalize negative behaviors from the sibling context to other contexts, increasing the risk for externalizing problems.

There is some empirical support for the association between sibling power balance and internalizing and externalizing problems. Whereas Ponzetti and James (1997) did not find significant correlations between sibling power balance and internalizing problems in a sample of White American college students, East and Khoo (2005) showed in an American non-White adolescent sample that more power from older sisters was linked to more sexual risk behavior in younger siblings. However, little is known about these processes in younger samples.

We do know that there are developmental changes in sibling power balance. Studies with European-American samples have found that power imbalance declines over time (Tucker et al., 2010), and that sibling relationships become more equal and less asymmetrical with increasing age (Buhrmester \& Furman, 1990). In other words, with increasing age, power imbalance may become less normative and therefore more problematic. This might mean that if there is indeed a large power imbalance between siblings, 
it could have stronger negative effects in older samples (in which power imbalance is nonnormative) than in younger samples. In the present study, we will therefore examine sibling power balance and its links with psychosocial adjustment in three different age groups: early childhood, late childhood, and adolescence.

Additionally, knowledge is lacking about the potentially different effects of sibling power balance on internalizing and externalizing problems. Effects may be stronger for externalizing problems (East \& Khoo, 2005) than for internalizing problems (Ponzetti \& James, 1997), but evidence is scarce. Therefore, in the current study we will examine the link between sibling power balance and internalizing as well as externalizing problems.

\section{Cross-Cultural Differences in Sibling Power Balance}

The few studies focusing on sibling power balance have mostly been conducted in Western societies. However, there may be differences between cultures in sibling relationship dynamics (Updegraff, McHale, Killoren, $\&$ Rodríguez, 2011). Some researchers even suggest that sibling relationships and culture are "inextricably tied" (Watson \& McGoldrick, 2011, p. 62).

Culture can be defined and operationalized in a number of ways. One dimension on which countries can be distinguished is individualism versus collectivism (Hofstede, Hofstede, \& Minkov, 2010). In more individualistic cultures, autonomy and independence of the individual are emphasized, whereas in more collectivistic cultures, relationships within and outside of the family are stressed (Peterson, Steinmetz, \& Wilson, 2005). Sibling relationships may be affected by the relative individualism/collectivism of a society (Cicirelli, 1994). Cultural norms may dictate expectations about sibling roles and responsibilities (especially for older siblings) more strongly in more collectivistic societies than in more individualistic societies, in which sibling roles and responsibilities are mostly voluntary (Cicirelli, 1994). This may result in cross-cultural differences in sibling power balance, with larger power imbalance between siblings in more collectivistic countries. However, the fact that there may be differences in power imbalance between cultures does not necessarily imply that the impact of sibling power imbalance on psychosocial functioning is different between cultures.

Whereas up to date there have been no cross-cultural examinations of the link between sibling power imbalance and psychosocial functioning, there have been some cross-ethnic or cross-cultural studies concerning warmth and conflict in the sibling relationship. These studies have generally shown more similarities than differences, especially concerning the link with problem behavior (Buist et al., 2014; Buist, Verhoeven, Hoksbergen, ter Laak, Watve, \& Paranjpe, 2016). So, for other dimensions 
of sibling relationship quality, there is more evidence for similarity across cultures. By taking a cross-cultural approach to sibling power balance and its concomitants, the present study adds greatly to our understanding of the role of cultural dynamics within the family system in order to develop family support programs. If the impact of sibling power imbalance is different in families with different cultural backgrounds, culture needs to be a factor in interventions.

\section{The Role of Age and Gender in Sibling Power Balance}

As mentioned earlier, age differences have been reported concerning sibling power balance. Additionally, age gap between the siblings has also been linked to sibling power balance, with greater power imbalance reported in dyads with a larger age gap (Buhrmester \& Furman, 1990). So, age and age gap between siblings should be taken into consideration when studying sibling power balance, which we did by controlling for age and age gap differences in the analyses. Additionally, because differences have been reported in sibling power balance concerning gender (Bigner, 1974) and birth order (Tucker et al., 2010; Volling, 2003), where possible we also controlled for gender and birth order in the analyses.

\section{The Present Study}

In the present study, we examined sibling power imbalance in three separate cross-cultural studies using different age groups. In the first study, using a between-country design, we compared a Turkish with a Dutch early childhood sample. In the second study, using a between-country design, we compared an Indian with a Dutch late childhood sample, and in the third study, using a within-country design, we compared two Dutch samples with either Moroccan or Dutch ethnicity. The choice for these countries was dictated by the main affiliation of the primary researchers. In all three studies, we first tested whether there were cross-cultural differences in sibling power balance. Second, in each study we examined whether there were cross-cultural differences in the link between sibling power imbalance and internalizing and externalizing problems.

Based on Cicirelli's work (1994), we expected more sibling power imbalance in more collectivistic (i.e., Turkish, Indian, and ethnic Moroccan; The Hofstede Centre, 2015) samples than in more individualistic (i.e., Dutch; The Hofstede Centre, 2015) samples. Additionally, based on earlier studies on sibling warmth and conflict (Buist et al., 2014, 2016), we did not expect differences between more collectivistic (i.e., Turkish, Indian, and ethnic Moroccan) and more individualistic (i.e., Dutch) samples concerning the effect of sibling power imbalance on internalizing and externalizing problem behavior. 


\section{Study 1: Early Childhood}

\section{Method.}

Procedure and participants. The first study was conducted in Turkey and the Netherlands. A total of 123 Turkish mothers (mean age 32.6 years) and 128 Dutch mothers (mean age 35.8 years) provided information about power imbalance in the sibling relationship and problem behavior of their child. Inclusion criteria were that mothers had at least two children, with one (target) child aged between four and six years old. In Turkey, mothers were recruited through preschools and in the Netherlands through elementary schools and personal networks of the research assistants. All mothers filled out the questionnaires at home. Dutch questionnaires were translated into Turkish with the use of a back-translation procedure. Dutch and Turkish mothers did not receive monetary compensation for their participation.

About half of the target children were the older sibling of the two. Of the Turkish children, $42.3 \%$ were male, and of the Dutch children $46.1 \%$. Distribution of sibling gender combinations was about even for both samples. Mean age of the children was 5.0 years for the Turkish children and 4.8 years for the Dutch children. Mean age for their siblings was 7.4 years for the Turkish sample and 5.1 years for the Dutch sample. Twins $(n=2)$ were excluded from the analyses. We tested for differences between the two samples concerning relevant background variables. The two samples did not differ significantly concerning child gender $\left[\chi^{2}(1)=0.52, p=0.47\right]$, sibling gender $\left[\chi^{2}(1)=1.52, p=0.22\right]$, nor birth order $\left[\chi^{2}(1)=1.22\right.$, $p=0.27]$. They did significantly differ concerning child age $(t=2.24, p<$ $0.05)$, sibling age $(t=4.29, p<0.001)$, and age gap between the siblings $(t=6.22, p<0.001)$. We therefore controlled for child and sibling age as well as age gap in the analyses.

\section{Measures.}

Sibling power imbalance. The Parent Report of Early Sibling relationship quality was used to assess power balance in the sibling relationship (PRE-SIB; Buist, 2013). Mothers reported on a 6-point Likert scale $(1=$ never to $6=$ extremely often) to what extent their child exerted power in the relationship with the sibling closest to him or her in age. A sample item of this 3-item scale is "My child acts bossy towards this brother/sister and orders him/her around." Higher scores indicate greater power imbalance between siblings. Reliability (Cronbach's alpha) was 0.62 for the Turkish sample and 0.82 for the Dutch sample. Because of this difference in reliability, we tested measurement equivalence. These analyses showed measurement invariance: constraining factor loadings across the samples did not significantly change model fit $\left[\Delta \chi^{2}(2)=1.23, p>.05\right]$.

Externalizing and internalizing problems. Child externalizing and internalizing problems were measured with the Child Behavior Checklist 1.5-5 years (Achenbach \& Rescorla, 2000). Mothers indicated on a 3-point scale [0 = not true (as far as you know), $1=$ somewhat or sometimes true, $2=$ 
very true or often true] whether their child showed certain behavior during the past 2 months. The Externalizing problems scale (24 items) measures parental perception of the degree of aggressive behavior and attention problems of their child. A sample item is "Gets in many fights." Reliability (Cronbach's alpha) was 0.81 for the Turkish and 0.88 for the Dutch sample. The Internalizing problems scale (36 items) measures parental perception of the degree of emotional reactivity, anxious/depressed and withdrawn behavior, and somatic complaints, for example "Withdrawn, doesn't get involved with others." Reliability was 0.89 for the Turkish and 0.82 for the Dutch sample.

\section{Results.}

Cross-Cultural Differences in Sibling Power Imbalance. To answer our first research question concerning cross-cultural difference in sibling power imbalance, we conducted an ANCOVA, in which we controlled for gender, child and sibling age, and age gap. Results showed that sibling power balance did not differ significantly between the Turkish $(M=2.78, S D=1.06)$ and Dutch children $(M=2.63, S D=0.83)$, with $F(1,243)=2.37, p=$ 0.13 .

Cross-Cultural Differences in Links Between Sibling Power Imbalance and Child Problem Behavior. To examine links between of sibling power imbalance and child externalizing and internalizing problem behavior, we applied path analyses in Mplus (version 7.11; Muthén \& Muthén, 1998-2012). Using observed variables, we formulated a multigroup model in which externalizing and internalizing problems were predicted by sibling power imbalance, while controlling for gender, child and sibling age, and age gap between siblings. The groups in this model were the Turkish and the Dutch sample.

We first tested a model in which paths were constrained to be equal for the Turkish and Dutch samples. Fit of this model was not good, with $\chi^{2}(2)=8.36, p=0.02$, root mean square error of approximation (RMSEA) $=0.16$, Tucker Lewis index (TLI) $=0.54$, comparative fit index (CFI) $=0.97$. Next, we checked whether releasing constraints between the two groups concerning paths improved fit. Indeed, releasing the constraint for the path between sibling power imbalance and externalizing problems (but not internalizing problems) significantly improved model fit: $\chi^{2}(1)=$ $0.14, p=0.71$, RMSEA $=0.00, \mathrm{TLI}=1.13, \mathrm{CFI}=1.00$. The difference in chi-square between these two models was significant: $\Delta \chi^{2}(1)=8.22, p<$ 0.01. Path estimations of this final model can be found in Table 6.1.

Our results indicate that for both samples, more sibling power imbalance was significantly linked to more internalizing problems. So, if children acted bossier towards their siblings, they also showed more internalizing problems according to their mothers. More sibling power imbalance was also significantly associated with more externalizing problems for both samples (see Table 6.1). However, for the Dutch sample, the link between sibling power imbalance and externalizing problems was significantly 


\section{Table 6.1. Effects of Sibling Power Imbalance on Externalizing and Internalizing Problems in Early-Childhood Samples}

\begin{tabular}{|c|c|c|c|c|c|c|}
\hline & \multicolumn{6}{|c|}{ Sibling Power Imbalance } \\
\hline & \multicolumn{3}{|c|}{ Turkey } & \multicolumn{3}{|c|}{ The Netherlands } \\
\hline & $B$ & SE (B) & $\beta$ & $B$ & SE (B) & $\beta$ \\
\hline Internalizing problems & 0.03 & 0.02 & $0.10^{*}$ & 0.03 & 0.02 & $0.16^{*}$ \\
\hline Externalizing problems & 0.06 & 0.02 & $0.18^{* *}$ & 0.14 & 0.03 & $0.41^{* * *}$ \\
\hline
\end{tabular}

Note: Fit final model $\chi^{2}(1)=0.14, p=0.71$, RMSEA $=0.00, T L I=1.13$, CFI $=1.00$. Covariates included in the model: gender, child and sibling age, and age gap.

$* p<0.05 . * * p<0.01 . * * * p<0.001$.

stronger $(\beta=0.41, p<0.001)$ than for the Turkish sample $(\beta=0.18$, $p<0.01)$.

\section{Study 2: Late Childhood}

\section{Method.}

Procedure and participants. The second study was conducted in India and the Netherlands. Self-report data were collected in schools from 124 Indian and 129 Dutch children. Children reported on sibling power imbalance and on their own problem behavior. The procedure and questionnaires for both samples was the same. Research assistants contacted elementary school principals to ask whether schools would participate in the study. If so, parents were given information about the study and the opportunity to refuse participation of their child by returning an answer slip. Data were collected in the classrooms under supervision of research assistants, who were available for questions about the study or the questionnaire. Dutch children filled out the questionnaires in Dutch, Indian children in English (they attended an English-language primary school). Dutch questionnaires were translated into English using a back-translation procedure. All children received a small gift (a pencil or eraser), whether they completed the questionnaire or not.

About half of the target children were the older sibling of the two. Of the Indian children, $48.4 \%$ were male, and of the Dutch children $52.7 \%$. For both samples, distribution of sibling gender combinations was about even. Mean age was 10.9 years for the Indian children and 11.0 years for the Dutch children. Mean age for their siblings was 10.6 years for the Indian sample and 11.3 years for the Dutch sample. Twins $(n=9)$ were excluded from the analyses.

We tested for differences between the two samples concerning relevant background variables. The two samples did not differ significantly concerning child gender $\left[\chi^{2}(1)=0.43, p=0.51\right]$, sibling gender $\left[\chi^{2}(1)=2.78\right.$, 
$p=0.10]$, birth order $\left[\chi^{2}(1)=1.40, p=0.24\right]$, child age $(t=1.21, p=$ $0.23)$, nor sibling age $(t=1.24, p=0.22)$. They did significantly differ concerning age gap between the siblings $(t=-4.85, p<0.001)$. We therefore controlled for age gap in the analyses.

\section{Measures.}

Sibling power imbalance. Power in the sibling relationship was measured with the Sibling Relationship Questionnaire (SRQ; Buhrmester \& Furman, 1990). Children were asked to fill out questions about the relationship with the sibling closest to them in age. Four items of the Dominance scale were used, rated along a 5-point Likert scale ranging from $1=$ hardly at all to $5=$ extremely much. A sample item is: "How much do you order this sibling around?". Higher scores indicate greater power imbalance between siblings. This scale had a Cronbach's alpha of .63 for the Indian and .79 for the Dutch sample. Again, analyses demonstrated measurement invariance: Model fit did not significantly change by constraining factor loadings across the samples $\left[\Delta \chi^{2}(3)=0.93, p>0.05\right]$.

Externalizing and internalizing problems. Externalizing and internalizing problem behavior was measured using the Nijmegen Problem Behavior List (NPBL Research version; De Bruyn, Scholte, \& Vermulst, 2005). The items were rated on a 5 -point scale $(1=$ does not apply to me at all to $5=$ applies to me very well). To assess externalizing problems, five items from the Aggression scale were used (e.g., "I fight a lot") which had a Cronbach's alpha of 0.68 for the Indian and 0.71 for the Dutch sample. To assess internalizing problems, four items from the Anxiety/Depression scale were used (e.g., "I worry a lot"). The Internalizing problems scale had a Cronbach's alpha of 0.58 for the Indian and 0.76 for the Dutch sample. This scale also showed measurement invariance: constraining factor loadings across the samples did not significantly change model fit $\left[\Delta \chi^{2}(3)=2.21, p>0.05\right]$.

\section{Results.}

Cross-cultural differences in sibling power imbalance. To answer our first research question concerning cross-cultural difference in sibling power imbalance, we conducted an ANCOVA, in which we controlled for gender, age, and age gap. Results showed that sibling power imbalance differed significantly between the Indian $(M=2.58, S D=0.89)$ and Dutch children $(M=2.01, S D=0.84)$, with $F(1,204)=24.27, p<0.001$. Indian children reported significantly more power imbalance in their sibling relationship.

Cross-cultural differences in links between sibling power imbalance and child problem behavior. To examine links between of sibling power imbalance and child externalizing and internalizing problem behavior, we formulated a multigroup model in which externalizing and internalizing problems were predicted by sibling power imbalance, while controlling for gender, age, and age gap between siblings. The groups in this model were the Indian and the Dutch sample. 


\section{Table 6.2. Effects of Sibling Power Imbalance on Externalizing and Internalizing Problems in Late-Childhood Samples}

\begin{tabular}{|c|c|c|c|c|c|c|}
\hline & \multicolumn{6}{|c|}{ Sibling Power Imbalance } \\
\hline & \multicolumn{3}{|c|}{ India } & \multicolumn{3}{|c|}{ The Netherlands } \\
\hline & $B$ & SE (B) & $\beta$ & $B$ & SE (B) & $\beta$ \\
\hline Internalizing problems & 0.20 & 0.06 & $0.22^{* *}$ & 0.20 & 0.06 & $0.23^{* *}$ \\
\hline Externalizing problems & 0.23 & 0.05 & $0.29^{* * *}$ & 0.23 & 0.05 & $0.32^{* * *}$ \\
\hline
\end{tabular}

Note: Fit final model $\chi^{2}(2)=1.13, p=0.58$, RMSEA $=0.00, \mathrm{TLI}=1.19, \mathrm{CFI}=1.00$. Covariates included in the model: gender, age, and age gap.

$* p<0.05 . * * p<0.01 . * * * p<0.001$.

We first tested a model in which paths were constrained to be equal for the Indian and Dutch samples. Fit of this model was excellent, with $\chi^{2}(2)=1.13, p=0.58$, RMSEA $=0.00, \mathrm{TLI}=1.19, \mathrm{CFI}=1.00$. Next, we checked whether releasing constraints between the two groups concerning paths improved fit. Releasing the constraint for the path between sibling power imbalance and externalizing problems or internalizing problems did not significantly improve model fit. Path estimations of the final (constrained) model can be found in Table 6.2.

Our results indicate that for both samples, more sibling power imbalance was significantly linked to more internalizing and externalizing problems (see Table 6.2). So, children who reported more power imbalance in their sibling relationship also reported more internalizing and externalizing problems.

\section{Study 3: Adolescence}

\section{Method.}

Procedure and participants. The third study was conducted in the Netherlands, with two adolescent samples with different ethnic backgrounds: ethnic Moroccan and ethnic Dutch. Participants were 165 ethnic Moroccan and a matched sample of 165 Dutch adolescents. Adolescents were considered ethnic Moroccan if the adolescents themselves or one of their parents were born in Morocco, and ethnic Dutch if the adolescents themselves as well as both parents were born in the Netherlands. Adolescents and their parents gave written informed consent, after which they were visited at their home by a trained interviewer to administer the questionnaires. Adolescents reported on their own problem behavior and on power in the sibling relationship. In the case of more than two adolescents, families were free to choose which two adolescents participated in the study. All participants filled out questionnaires in Dutch. All participating families received a monetary compensation of $€ 100$. 
Of the ethnic Moroccan adolescents, $47.3 \%$ were male and mean age was 15.3 years. Of the ethnic Dutch adolescents, $46.7 \%$ were male and mean age was 15.2 years. For both samples, distribution of sibling gender combinations was about even, with a slight overrepresentation of sister pairs (34.3\%). None of the sibling pairs were twins. We tested for differences in background variables. No differences were found for adolescent gender $\left[\chi^{2}(1)=0.01, p=0.91\right]$, nor sibling gender $\left[\chi^{2}(1)=0.90, p=0.34\right]$, but $t-$ tests showed that ethnic Moroccan adolescents were significantly older than ethnic Dutch adolescents $(t=2.19, p<0.05)$. We therefore controlled for age in the analyses.

\section{Measures.}

Sibling power imbalance. Sibling power was measured by six items from the Network of Relationship Inventory (NRI: Furman \& Buhrmester, 1985). Adolescents rated the degree of sibling domination on a 5-point Likert scale ( 1 = little to none and $5=$ could not be more). A sample item is: "How often does your brother/sister tell you what to do?". Higher scores indicate greater power imbalance between siblings. Cronbach's alpha was .90 for the Moroccan and .93 for the Dutch adolescents.

Externalizing and internalizing problems. Externalizing problems were measured with the 30-item Externalizing problems scale of the Youth Self Report (YSR: Achenbach, 1991; Verhulst, van der Ende, \& Koot, 1997). Adolescents indicated their externalizing problems over the past 6 months on a 3-point Likert scale $(1=$ not true, $2=$ somewhat or sometimes true, and 3 = very true or often true). A sample items is: "I fight a lot." Sum scores were calculated, with higher scores indicating more externalizing problems. Cronbach's alpha was .94 for the Moroccan and .88 for the Dutch adolescents.

Adolescent anxiety was measured with 18 items from the Screen for Child Anxiety Related Emotional Disorders (SCARED; Birmaher et al., 1997; Hale, Raaijmakers, Muris, \& Meeus, 2005). Items from the social anxiety, generalized anxiety, and separation anxiety scales were summed to reflect total anxiety. Adolescents rated their anxiety on a 3-point Likert scale $(1=$ almost never, $2=$ sometimes, and $3=$ often $)$. A sample item is: "I am afraid to be home alone." Cronbach's alpha was 0.92 for Moroccan and 0.95 for Dutch adolescents.

The Reynolds Adolescent Depression Scale-2nd edition (RADS-2; Reynolds, 2000) was used to measure adolescent depression. All 30 items were summed to reflect total depression. Adolescent rated their depressed feelings on a 4-point Likert scale $(1=$ almost never and $4=$ most of the time $)$. A sample item is: "I feel like nobody cares about me." Cronbach's alpha was 0.94 for Moroccan and 0.95 for Dutch adolescents.

\section{Results.}

Cross-cultural differences in sibling power imbalance. To answer our first research question concerning cross-cultural difference in sibling power imbalance, we conducted an ANCOVA, in which we controlled for age and 


\section{Table 6.3. Effects of Sibling Power Imbalance on Externalizing and Internalizing Problems in Adolescent Samples}

\begin{tabular}{|c|c|c|c|c|c|c|}
\hline & \multicolumn{6}{|c|}{ Sibling Power Imbalance } \\
\hline & \multicolumn{3}{|c|}{ Moroccan } & \multicolumn{3}{|c|}{ Dutch } \\
\hline & $B$ & SE (B) & $\beta$ & $B$ & SE (B) & $\beta$ \\
\hline Externalizing problems & 1.83 & 0.63 & $0.17^{* *}$ & 1.83 & 0.63 & $0.19^{* *}$ \\
\hline Anxiety & 3.03 & 0.83 & $0.25^{* * *}$ & 3.03 & 0.83 & $0.21^{* * *}$ \\
\hline Depression & 2.90 & 0.88 & $0.22^{* *}$ & 2.90 & 0.88 & $0.18^{* *}$ \\
\hline
\end{tabular}

Note: Fit final model $\chi^{2}(3)=0.74, p=0.86$, RMSEA $=0.00, \mathrm{TLI}=1.07, \mathrm{CFI}=1.00$. Covariates included in the model: gender and age.

$* p<0.05 . * * p<0.01 . * * * p<0.001$.

gender. Results showed that sibling power imbalance did not differ significantly between the Moroccan $(M=2.07, S D=0.84)$ and Dutch adolescents $(M=2.06, S D=0.81)$, with $F(1,242)=0.00, p=.99$.

Cross-cultural differences in links between sibling power imbalance and adolescent problem behavior. To examine links between sibling power imbalance and adolescent externalizing and internalizing problems, we formulated a multi-group model in which externalizing problems, anxiety, and depression were predicted by sibling power imbalance, while controlling for age and gender of the adolescent. The groups in this model were the Moroccan and the Dutch sample.

We first tested a model in which paths were constrained to be equal for the Moroccan and Dutch samples. Fit of this model was excellent, with $\chi^{2}(3)=0.74, p=0.86$, RMSEA $=0.00, \mathrm{TLI}=1.07, \mathrm{CFI}=1.00$. Next, we checked whether releasing constraints between the two groups concerning paths improved fit. Releasing the constraint for the path between sibling power imbalance and externalizing problems, anxiety or depression did not significantly improve model fit. Path estimations of the final (constrained) model can be found in Table 6.3.

Our results indicate that for both samples, more sibling power imbalance was significantly linked to more internalizing and externalizing problems (see Table 6.3). So, adolescents who reported more power from and dominance by their sibling also reported more internalizing and externalizing problems.

\section{Discussion}

The aims of the present study were (1) to examine potential cross-cultural differences in sibling power imbalance in three different studies, with samples from early childhood, late childhood and adolescence and (2) to 
compare the associations between sibling power imbalance and problem behavior in these different samples.

Based on Cicirelli (1994), who described large differences between different countries in sibling relationships, we had expected differences between the more individualistic and more collectivistic countries in sibling power imbalance. This hypothesis was only confirmed for the late-childhood sample: Indian children reported that they experienced more power imbalance in the sibling relationship than their Dutch counterparts. In the early-childhood and adolescent samples, we found no cross-cultural differences in sibling power imbalance. So, for children in early childhood and adolescence, similar levels of sibling power imbalance were reported across cultures, either by their mothers or by adolescents themselves.

We hypothesized no differences between more individualistic countries and more collectivistic countries concerning the effect of sibling power imbalance on problem behavior, based on earlier work on sibling warmth and conflict (Buist et al., 2014, 2016). This hypothesis was confirmed for the late childhood and adolescent samples, but not the early childhood sample: the link between sibling power imbalance and externalizing problems (as reported by mothers) was stronger for Dutch versus Turkish children in early childhood. For internalizing problems, this was not the case. It may be that young children who are bossy towards their sibling also behave more aggressively toward other children (Abuhatoum \& Howe, 2013). The difference may also be due to cross-cultural differences in the interpretation of behavior (Stevens et al., 2003). In Turkey, mothers may not consider bossy behavior towards other children as aggression and may be proud of their child for being able to stand up for him/herself. For the late-childhood and adolescent samples no significant differences in associations were found.

Overall, we found few cross-cultural differences. The consistency of findings across the three studies suggest that cross-cultural variations in sibling power imbalance and their impact on problem behavior may actually be small. Sibling power imbalance appears to be operating similarly across more individualistic and more collectivistic cultures. Kagitcibasi's model of family change (1996, 2006, 2007) emphasizes that viewing Western cultures as individualistic with a strong emphasis on total independence and non-Western cultures as collectivistic stressing total interdependence may not apply to today's families. According to Kagitcibasi (2005), families today, particularly ethnic minorities living in Europe and North America, can be understood better with a perspective that emphasizes autonomous related selves, indicating that families can be materially separated yet psychologically interdependent. Kagitcibasi's model indicates that dualistic perspectives on the cultures limit our understanding of families and dynamics of healthy human development. In fact, research findings demonstrating more similarities across cultures (e.g., Buist et al., 2014, 2016) as well as our own results may just illustrate the need for a more comprehensive approach. However, despite this need for a more comprehensive approach to 
understand sibling relationships and particularly sibling power imbalance, studies that include samples with various cultural and/or ethnical backgrounds have been scarce. Our study may be an important starting point in understanding how cultural norms dictating sibling roles, responsibilities, and expectations within different cultures affect how perceived power imbalance within sibling relationships influence psychosocial functioning of children and adolescents.

Across all four cultures represented in the current study, more power imbalance in the sibling relationship (either more power for the child/adolescent or for his or her sibling) is linked to more internalizing and externalizing problems. Whereas sibling power imbalance is sometimes seen as natural and normative, our results indicate that they are not perceived as such by parents and children. Across different age groups and different cultural groups, a power imbalance within the sibling relationship, with one sibling dominating the other, seems to have a detrimental effect on anxiety, depression, and aggression. This appears to be the case regardless of who is dominant: the target children themselves (early childhood sample), their siblings (adolescent sample), or a combination of both (late childhood sample). Despite inherent age-related power differences between siblings because of birth order, children and adolescents seem to function best if the power imbalance between themselves and their sibling is limited. We found no clear differences between internalizing and externalizing problems, which is only partly consistent with earlier studies (East \& Khoo, 2005; Ponzetti \& James, 1997). Studies with older samples did not find a significant link between sibling power imbalance and internalizing problems (Ponzetti \& James, 1997), whereas we did, across all samples and ages. Whereas we did not statistically test for age-related differences, our results did not suggest differences between the three age groups. It may be that sibling power imbalance is more influential for children than for adults, but more longitudinal research is needed to clarify these over-time patterns.

Strengths, Limitations, and Future Directions. Several limitations are relevant for all three of the studies. Only cross-sectional data were used, precluding causal interpretations. Whereas it may be that problem behavior is affected by sibling power imbalance, the reverse pattern is also possible: aggressive or depressed children behave less empathically and more bossily in interactions with his or her sibling. Longitudinal research is needed to examine these potential reciprocal patterns.

Comparability of the samples and measures is always an important issue in cross-cultural research. It is possible that the samples in the studies are not representative for the entire population. This may contribute to the lack of cross-cultural differences found in all three studies. Adding a measure of adherence to cultural norms could address some of these issues. Comparability of measures was checked by examining measurement invariance. Our findings suggest that there were no significant differences 
between countries concerning the interpretation of measurements by our participants.

Additionally, conclusions are based on single-informant self-report data of mothers or children. Their interpretation of sibling behavior may be biased. Observation of child behavior within and outside the sibling relationship may provide valuable insights into these processes. Also, it is noteworthy that reliabilities of our measures were not always satisfactory. The relatively small sample sizes could also limit the power of our analyses (precluding analyses focusing on potential moderating factors such as gender or birth order), as well as the generalizability of our findings. It is important to take these limitations into consideration when interpreting our results.

The design of the study, with three studies comparing four countries in three age groups can be considered a strength as well as a limitation. Whereas this design allows us to examine cross-cultural as well as agerelated sibling patterns, these two are confounded. Because of this overlap, it is difficult to determine whether our results can be explained by differences between the specific countries or by age differences between the studies.

Notwithstanding these limitations, our study addresses a gap in knowledge concerning sibling power imbalance. Sibling power imbalance is already an understudied subject, but cross-cultural studies concerning sibling power imbalance have been extremely scarce. Our study has contributed to existing knowledge about cross-cultural similarities in sibling power imbalance and its link with child and adolescent functioning. Additionally, we have studied sibling power imbalance and its concomitants in three different age groups to examine age-related patterns.

\section{Conclusions and Implications}

Across countries and ethnicities and across age groups, more sibling power imbalance was linked to more internalizing and externalizing problems. When replicated, these insights may help health-care professionals with fine-tuning existing or developing new interventions concerning sibling power imbalance. First, it seems fruitful to focus on sibling power imbalance when children or adolescents are exhibiting internalizing or externalizing problems. Second, when addressing sibling power imbalance, it is important to realize that culture does not seem to play a large role in these processes.

\section{Author Note}

The data for Study 3 were collected as part of the Research on Adolescents Development And Relationships (RADAR) research project, supported by main grants from the Netherlands Organization for Scientific Research 
(GB-MAGW 480-03-005), and Stichting Achmea Slachtoffer en Samenleving (SASS), and various other grants from the Netherlands Organization for Scientific Research, the Vrije Universiteit Amsterdam, and Utrecht University. The authors would like to express their gratitude to the children, adolescents, and mothers that participated in the studies and to the research assistants for their valuable efforts in data collection. A special thanks to research assistants Lisette Dragt and Jessie Hillekens for their help during the preliminary phase of this study.

\section{References}

Abuhatoum, S., \& Howe, N. (2013). Power in sibling conflict during early and middle childhood. Social Development, 22, 738-754. https://doi.org/10.1111/sode.12021

Achenbach, T. M. (1991). Manual for the Youth Self-Report and 1991 Profile. Burlington, VT: University of Vermont, Department of Psychiatry.

Achenbach, T. M., \& Rescorla, L. A. (2000). Manual for the ASEBA Preschool Forms \& Profiles. Burlington, VT: University of Vermont, Research Center for Children, Youth, \& Families.

Bigner, J. J. (1974). Second borns' discrimination of sibling role concepts. Developmental Psychology, 10, 564-573. https://doi.org/10.1037/h0036599

Birmaher, B., Khetarpal, S., Brent, D., Cully, M., Balach, L., Kaufman, J., \& McKenzie Neer, S. (1997). The Screen for Child Anxiety Related Emotional Disorders (SCARED): Scale construction and psychometric characteristics. Journal of the American Academy of Child and Adolescent Psychiatry, 36, 545-553. https://doi.org/10.1097/00004583-199704000-00018

Buhrmester, D. (1992). In F. Boer \& J. Dunn (Eds.), Children's sibling relationships: Developmental and clinical issues (pp. 19-40). Hillsdale, NJ: Erlbaum.

Buhrmester, D., \& Furman, W. (1990). Perceptions of sibling relationships during middle childhood and adolescence. Child Development, 61, 1387-1398. https://doi.org/10.2307/1130750

Buist, K. L. (2013). The parent report of early sibling relationship quality (PRE-SIB). Unpublished manuscript, Utrecht University, Utrecht.

Buist, K. L., Deković, M., \& Prinzie, P. (2013). Sibling relationship quality and psychopathology of children and adolescents: A meta-analysis. Clinical Psychology Review, 33, 97-106. https://doi.org/10.1013/j.cpr.2012.10.007

Buist, K. L., Paalman, C. H., Branje, S. J. T., Deković, M., Reitz, E., Verhoeven, M., . . Hale, W. W. III. (2014). Longitudinal effects of sibling relationship quality on adolescent problem behavior: A cross-ethnic comparison. Cultural Diversity and Minority Psychology, 20, 266-275. https://doi.org/10.1037/a0033675

Buist, K. L., Verhoeven, M., Hoksbergen, R., ter Laak, J., \& Watve, S., Paranjpe, A., (2016). Associations of perceived sibling and parent-child relationship quality with internalizing and externalizing problems: Comparing Indian and Dutch early adolescents. Journal of Early Adolescence. Advance online publication. https://doi.org/10.1177/0272431616653473

Caspi, J. (2011). Future directions for sibling research, practice, and theory. In J. Caspi (Ed.), Sibling development: Implications for mental health practitioners (pp. 377-390). New York, NY: Springer.

Cicirelli, V. G. (1994). Sibling relationships in cross-cultural perspective. Journal of Marriage $\&$ the Family, 56, 7-20. https://doi.org/10.2307/352697

De Bruyn, E. E. J., Scholte, R. H. J., \& Vermulst, A. A. (2005). Psychometric analyses of the Nijmegen problem behavior list (NPBL): A research instrument for assessing problem 
behavior in community samples using self- and other reports of adolescents and parents. Nijmegen, The Netherlands: Institute of Family and Child Studies, Radboud University Nijmegen.

Dirks, M. A., Persram, R., Recchia, H. E., \& Howe, N. (2015). Sibling relationships as sources of risk and resilience in the development and maintenance of internalizing and externalizing problems during childhood and adolescence. Clinical Psychology Review, 42, 145-155. https://doi.org/10.1016/j.cpr.2015.07.003

East, P. L., \& Khoo, S. T. (2005). Longitudinal pathways linking family factors and sibling relationship qualities to adolescent substance use and sexual risk behaviors. Journal of Family Psychology, 19, 571-580. https://doi.org/10.1037/0893-3200.19.4.571

Furman, W., \& Buhrmester, D. (1985). Children's perceptions of the personal relationships in their social networks. Developmental Psychology, 21, 1016-1024. https://doi.org/10.1037/0012-1649.21.6.1016

Hale, W. W. III, Raaijmakers, Q., Muris, P., \& Meeus, W. (2005). Psychometric properties of the Screen for Child Anxiety Related Emotional Disorders (SCARED) in the general adolescent population. Journal of the American Academy of Child \& Adolescent Psychiatry, 44, 283-290. https://doi.org/10.1097/00004583-200503000-00013

Hofstede, G., Hofstede, G. J., \& Minkov, M. (2010). Cultures and organizations: Software of the mind (3rd ed.). New York, NY: McGraw-Hill.

Kagitcibasi, C. (1996). Family and human development across cultures: A view from the other side. Hillsdale, NJ: Erlbaum.

Kagitcibasi, C. (2005). Autonomy and relatedness in cultural context implications for self and family. Journal of Cross-Cultural Psychology, 36, 403-422. https://doi.org/10.1177/0022022105275959

Kagitcibasi, C. (2006). Theoretical perspectives on family change. In J. Georgas, J. W. Berry, FJ.R. van de Vijer, C. Kagitcibasi, \& Y. H. Poortinga (Eds.), Families across cultures: A 30-nation psychological study (pp. 72-89). Cambridge, England: Cambridge University Press.

Kagitcibasi, C. (2007). Family, self, and human development across cultures: Theory and applications, (2nd ed.). Hillsdale, NJ: Erlbaum.

Muthén, L. K., \& Muthén, B. O. (1998-2012). Mplus user's guide (7th ed.). Los Angeles, CA: Muthén \& Muthén.

Peterson, G. W., Steinmetz, S. K., \& Wilson, S. M. (2005). Persisting issues in cultural and cross-cultural parent-youth relations. Marriage and Family Review, 36, 229-240. https://doi.org/10.1300/J002v36n03_11

Ponzetti, J. J., \& James, C. M. (1997). Loneliness and sibling relationships. Journal of Social Behavior and Personality, 12, 103-112.

Reynolds, W. M. (2000). Reynolds Adolescent Depression Scale-2nd edition (RADS-2). Professional manual. Lutz, FL: Psychological Assessment Resources.

Stevens, G. W., Pels, T., Bengi-Arslan, L., Verhulst, F. C., Vollebergh, W. A., \& Crijnen, A. A. (2003). Parent, teacher and self-reported problem behavior in The Netherlands: Comparing Moroccan immigrant with Dutch and with Turkish immigrant children and adolescents. Social Psychiatry and Psychiatric Epidemiology, 38, 576-585. https://doi.org/10.1007/s00127-003-0677-5

The Hofstede Centre. (2015). Country comparison: What about the Netherlands? Retrieved from http://geert-hofstede.com/netherlands.html

Tucker, C. J., Updegraff, K., \& Baril, M. E. (2010). Who's the boss? Patterns of control in adolescents' sibling relationships. Family Relations, 59, 520-532. https://doi.org/10.1111/j.1741-3729.2010.00620.x

Updegraff, K. A., McHale, S. M., Killoren, S. E., \& Rodriguez, S. A. (2011). Cultural variations in sibling relationships. In J. Caspi (Ed.), Sibling development: Implications for mental health practitioners (pp. 83-105). New York, NY: Springer. 
Verhulst, F. C., Van der Ende, J., \& Koot, H. M. (1997). Handleiding voor de Youth Self-Report (YSR) [Manual for the Youth Self-Report (YSR)]. Rotterdam, The Netherlands: Department of Child and Adolescent Psychiatry/Psychology, Erasmus University Medical Center-Sophia Children's Hospital.

Volling, B. L. (2003). Sibling relationships. In: M. H. Bornstein, L. Davidson, C. L. M. Keyes, \& K. A. Moore (Eds.), Well-being: Positive development across the life course (pp. 205-220). Mahwah, NJ: Erlbaum.

Watson, M. F., \& McGoldrick, M. (2011). Practice with siblings in a cultural context. In J. Caspi (Ed.), Sibling development: Implications for mental health practitioners (pp. 59-81). New York, NY: Springer Publishing Co.

KirSten L. BUist is an Assistant Professor in the Department of Clinical Child and Family Studies at Utrecht University, the Netherlands. She received her $\mathrm{PhD}$ in family psychology from Utrecht University in 2002. Her research interests focus on the link between the family system (in particular parent-child and sibling relationships) and child and adolescent psychosocial adjustment.

Aysegül MetINDOGAn works at Bogazici University School of Education, teaching developmental psychology and research methods courses and training master students in clinical psychology \& couples and family therapy. She conducts research on child development, parenting, fathering, gender and marital relationships and received her masters and doctoral degree on child and family studies.

SELMA ÇOBAN is a 2nd year graduate student in Clinical Psychology at Bilgi University. She is currently completing her clinical practicum in the Adult Track at the Bilgi University Psychological Counselling Unit. She also works part-time as a preschool principal. She received a BA in a double major program in Guidance and Psychological Counselling and in Preschool Education from Boğaziçi University in 2014. Her current research focuses on emotion regulation, somatization and the mind-body relationship in psychotherapy.

Sujala WATVE works as Secretary for Jnana Prabodhini Samshodhan Sanstha, is as a member of Research Advisory Boards and an Institutional Ethics committee as well as being National Supervisory Psychologist for Mensa India. She received her Ph.D from Pune University, India. She has special interest in identification, nurture and developing support system for High Ability individuals.

Analpa PaRAnjpe received her PhD in Psychology and has worked as Head researcher and visiting faculty at Jnana Prabodhini's Institute of Psychology in Pune (India). She has extensive experience as a psychologist, specializing in guidance for children with special needs and their parents and was director of the Dr. Anjali Morris Foundation. She is currently Principal of the Special Education department at the Indian School in Muscat (Sultanate of Oman). 
HaNs M. KоOT, PhD, RN, is emeritus professor of developmental psychology and developmental psychopathology in the Department of Clinical, Neuro and Developmental Psychology at Vrije Universiteit Amsterdam, The Netherlands. His research interests include emotional and behavioral development, developmental psychopathology, prevention in mental health care, quality of life, intellectual disability, and autism.

POL VAN LIER is a professor of Developmental Psychology at Erasmus University Rotterdam. He received his PhD in 2002 at Erasmus Medical Center. His research focuses on the influence of social experiences with parents, peers, and teachers on the development of psychopathology in children, as well as stressand self-regulation as potential mediators of that influence.

SUSAN J. T. BRANJE is a professor of Adolescent Development and Socialization at Department of Youth and Family, Utrecht University, The Netherlands. She received her PhD cum laude at the Radboud University Nijmegen in 2003. Her work focuses on understanding the developmental changes in adolescents' individual characteristics and relationships with parents, siblings, friends, and romantic partners, as well as the associations with development of adolescent adjustment.

Wim H. J. MeEus, PhD, is Professor of Adolescent Development at Utrecht University, and Professor of Developmental Psychology at Tilburg University, The Netherlands. He received his doctorate in Social Psychology from Utrecht University, The Netherlands. His major research interests include identity and personality development, personal relationships, and psychosocial problems in adolescence. 